\title{
Synthesis of the C12-C19 Fragment of (+)-Peloruside A through a Diastereomer-Discriminating RCM Reaction
}

\author{
Emmanuel Roulland and Mikhail S. Ermolenko* \\ Institut de Chimie des Substances Naturelles, CNRS, \\ avenue de la Terrasse, 91198 Gif-Sur-Yvette, France. \\ mikhail.ermolenko@icsn.cnrs-gif.fr
}

\section{Supporting Information}

Table of Contents:

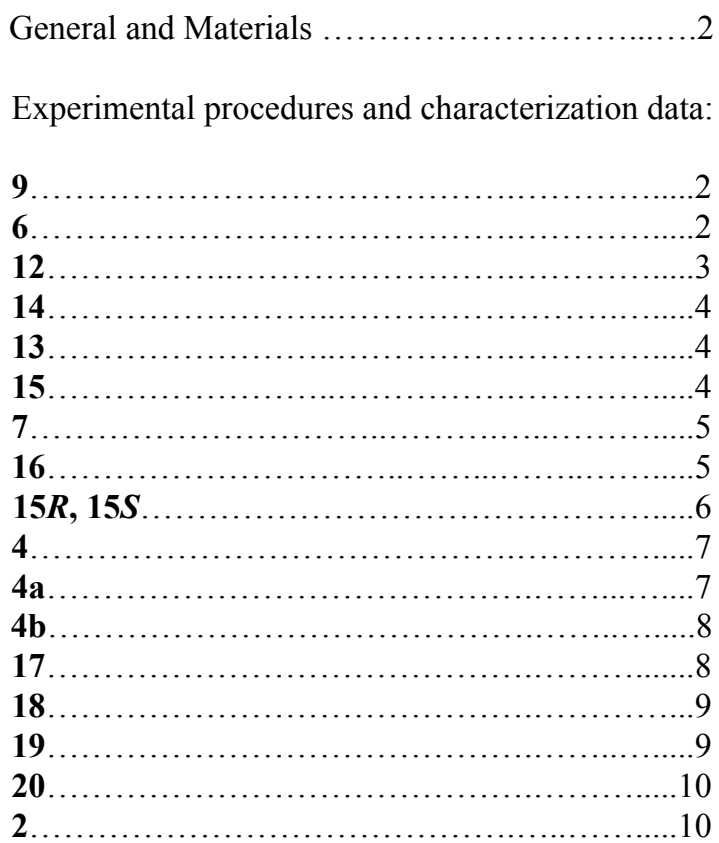

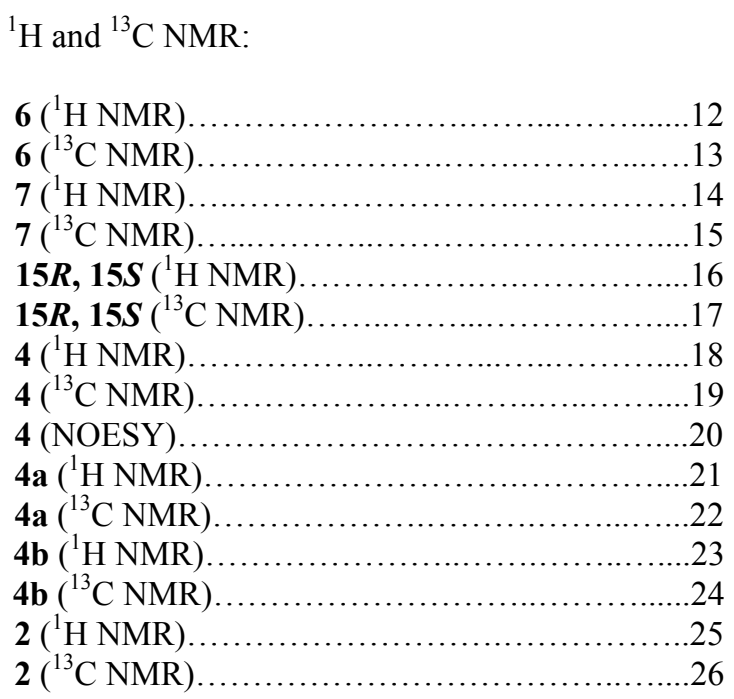




\section{Experimental section}

General Experimental procedures. NMR spectra were recorded (BRUKER 300, 400 or 500 $\mathrm{MHz},{ }^{1} \mathrm{H}$ and 75 or $100 \mathrm{MHz},{ }^{13} \mathrm{C}$ ) in $\mathrm{CDCl}_{3}$ (which also provided the lock signal at $\delta=7.26$ ppm, ${ }^{1} \mathrm{H}$ and $\delta=77.16 \mathrm{ppm},{ }^{13} \mathrm{C}$ ). IR spectra were recorded on a Perkin Elmer FT-IR system using diamond window Dura SamplIR II. Mass spectra were determined with Micromass LCT. Optical rotations were measured on a JASCO P-1010 polarimeter. Silica gel 60 (35-70 $\mu \mathrm{m})$ was used for flash chromatography. TLC plates (Merck $60 \mathrm{~F}_{254}$ aluminum sheets) were rendered visible by ultraviolet and/or spraying with phosphomolybdic acid (5\% in ethanol) or with vanillin (1\%) and sulphuric acid (5\%) in ethanol followed by heating. THF and ether were distilled under argon from sodium/benzophenone prior to use. Dichloromethane was distilled from $\mathrm{CaH}_{2}$ under argon prior to use. Second generation Grubbs catalyst was purchassed at STREM (ref 44-7770 lot\# B7840042) and (S)-propylene oxide 8 was prepared by the procedure described in ref 5 .

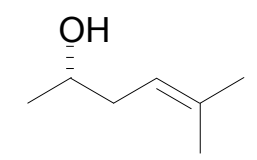

\section{5-Methyl-hex-4-en-2-ol (9):}

A solution of 2-methyl-1-propenylmagnesium bromide in THF (0.5 M, $297 \mathrm{~mL}, 148.6 \mathrm{mmol})$ was cooled to $-35^{\circ} \mathrm{C}$ under an argon atmosphere. Copper iodide $(1.29 \mathrm{~g}, 6.75 \mathrm{mmol}, 5 \mathrm{~mol} \%)$ was introduced followed $15 \mathrm{~min}$ later by $(S)$-propylene oxide 8 (7.85 g, $135.1 \mathrm{mmol})$ which was added dropwise over a $20 \mathrm{~min}$ period. After $30 \mathrm{~min}$ of stirring, the flask was removed from the cold bath and allowed to reach room temperature. The reaction was quenched $45 \mathrm{~min}$ later by pouring the reaction mixture into a separatory funnel containing $500 \mathrm{~mL}$ of brine and $12.4 \mathrm{~mL}$ of $12 \mathrm{~N}$ hydrochloric acid solution. After extraction with EtOAc, the organic phase was dried over anhydrous $\mathrm{Na}_{2} \mathrm{SO}_{4}$ and the solvent was removed under vacuum. The alcohol was purified by distillation $\left(35^{\circ} \mathrm{C}, 0.2 \mathrm{mmHg}\right)$ affording 9 as a colorless oil (13.30 g, $\left.87 \%\right)$.

$[\alpha]^{23}{ }_{\mathrm{D}}+18.3\left(c\right.$ 1.08, $\mathrm{CH}_{2} \mathrm{Cl}_{2}$ ); $\mathrm{R} f 0.43$ (heptane/EtOAc, 1:1); IR (film) 3338, 2967, 2914, $1449,1374,1119,1076 \mathrm{~cm}^{-1} ;{ }^{1} \mathrm{H}$ NMR $\left(300 \mathrm{MHz}, \mathrm{CDCl}_{3}\right) \delta_{\mathrm{H}} 1.19(\mathrm{~d}, 3 \mathrm{H}, J=6.2 \mathrm{~Hz}), 1.64$ $(\mathrm{s}, 3 \mathrm{H}), 1.74(\mathrm{~d}, 3 \mathrm{H}, J=1.7 \mathrm{~Hz}), 1.88(\mathrm{~s}, 1 \mathrm{H}, \underline{\mathrm{OH}}), 2.15(\mathrm{~m}, 2 \mathrm{H}), 3.79(\mathrm{~m}, 1 \mathrm{H}), 5.17(\mathrm{~m}, 1 \mathrm{H})$; ${ }^{13} \mathrm{C} \mathrm{NMR}\left(75 \mathrm{MHz}, \mathrm{CDCl}_{3}\right) \delta_{\mathrm{C}} 18.0,22.8,26.0,38.1,68.0,120.3,135.0$.

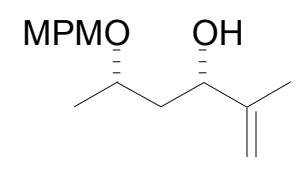

\section{5-(4-Methoxy-benzyloxy)-2-methyl-hex-1-en-3-ol (6):}

The alcohol 9 (1.137 g, $8.76 \mathrm{mmol})$ was dissolved in anhydrous $\mathrm{CH}_{2} \mathrm{Cl}_{2}(35 \mathrm{~mL})$ under an argon atmosphere. This solution was cooled to $-25^{\circ} \mathrm{C}$, then $\mathrm{VO}(\mathrm{acac})_{2}(46.2 \mathrm{mg}, 0.17 \mathrm{mmol}$, $2 \mathrm{~mol} \%)$ was added followed by a dropwise addition of tert $-\mathrm{BuO}_{2} \mathrm{H}(2.07 \mathrm{~mL}$ of a $5.5 \mathrm{M}$ solution in decane, $11.38 \mathrm{mmol}$ ). After 3 days of stirring at $-25{ }^{\circ} \mathrm{C}$ the flask was removed from the cold bath, the dichloromethane was carefully distilled off under atmospheric pressure. The residue was then taken into anhydrous THF $(35 \mathrm{~mL})$ and cooled to $0{ }^{\circ} \mathrm{C}$ under an argon atmosphere. Sodium hydride (1.05 g, 60\% in mineral oil, washed with pentane, 26.3 mmol) was added followed $20 \mathrm{~min}$ later by $\mathrm{Bu}_{4} \mathrm{NI}(323 \mathrm{mg}, 0.88 \mathrm{mmol}, 10 \mathrm{~mol} \%)$ and 4-methoxybenzyl chloride $(2.38 \mathrm{~mL}, 17.51 \mathrm{mmol})$. After $24 \mathrm{~h}$ of stirring at room temperature, the reaction mixture was poured into a separatory funnel containing $200 \mathrm{~mL}$ of a saturated aqueous $\mathrm{NaHCO}_{3}$ solution. After extraction with EtOAc, the organic phase was dried over anhydrous $\mathrm{Na}_{2} \mathrm{SO}_{4}$ and the solvent was removed under vacuum affording epoxide $\mathbf{1 1}$ as an oil. 
Crude epoxide 11 was transferred into a $i-\mathrm{Pr}_{2} \mathrm{NMgBr}$ THF solution (prepared in advance from a $3 \mathrm{M} \mathrm{MeMgBr}$ in ether $(8.76 \mathrm{~mL}, 26.3 \mathrm{mmol})$ and diisopropylamine $(3.68 \mathrm{~mL}, 26.3 \mathrm{mmol})$ in $25 \mathrm{~mL}$ of anhydrous THF) and the mixture was refluxed during $2 \mathrm{~h}$. The reaction was quenched by cautious pouring in a saturated aqueous $\mathrm{NH}_{4} \mathrm{Cl}$ solution. After extraction with EtOAc, the organic phase was dried over anhydrous $\mathrm{Na}_{2} \mathrm{SO}_{4}$ and the solvent was removed under vacuum. The residue was purified by flash chromatography (heptane/EtOAc, 5:1) affording the allylic alcohol $6(1.796 \mathrm{~g}, 82 \%)$ as a yellow oil. This oil was purified by distillation using a BÜCHI glass oven $\left(120^{\circ} \mathrm{C}, 4.10^{-2} \mathrm{mmHg}\right)$ affording pure alcohol 6 as a colorless oil.

$[\alpha]_{\mathrm{D}}^{23}+48.5$ (c 2.1, $\mathrm{CHCl}_{3}$ ); $\mathrm{R} f 0.21$ (heptane/EtOAc, 2:1); IR (film) 3457, 2968, 2935, 2869, 2837, 1651, 1613, 1586, 1514, $1249 \mathrm{~cm}^{-1} ;{ }^{1} \mathrm{H}$ NMR $\left(300 \mathrm{MHz}, \mathrm{CDCl}_{3}\right) \delta_{\mathrm{H}} 1.25(\mathrm{~d}, 3 \mathrm{H}, J=6.1$ $\mathrm{Hz}), 1.60-1.82(\mathrm{~m}, 5 \mathrm{H}), 3.65(\mathrm{~s}, 1 \mathrm{H}, \underline{\mathrm{OH}}), 3.81(\mathrm{~m}, 4 \mathrm{H}), 4.22(\mathrm{~d}, 1 \mathrm{H}, J=8.3 \mathrm{~Hz}), 4.36(\mathrm{~d}, 1 \mathrm{H}$, $J=10.9 \mathrm{~Hz}), 4.60(\mathrm{~d}, 1 \mathrm{H}, J=10.9 \mathrm{~Hz}), 4.79(\mathrm{t}, 1 \mathrm{H}, J=1.4 \mathrm{~Hz}), 4.97(\mathrm{t}, 1 \mathrm{H}, J=0.9 \mathrm{~Hz})$, $6.88(\mathrm{~d}, 2 \mathrm{H}, J=8.7 \mathrm{~Hz}), 7.26(\mathrm{~d}, 2 \mathrm{H}, J=8.6 \mathrm{~Hz}) ;{ }^{13} \mathrm{C}$ NMR $\left(75 \mathrm{MHz}, \mathrm{CDCl}_{3}\right) \delta_{\mathrm{C}} 199.1$, $20.9,43.8,56.5,71.3,76.7,76.8,111.6,115.1,130.7,131.3,148.3,160.5 ; \mathrm{MS}$ (TOF) $\mathrm{m} / \mathrm{z}$ $273.1\left([\mathrm{M}+\mathrm{Na}]^{+}\right)$; Anal. calcd for $\mathrm{C}_{15} \mathrm{H}_{22} \mathrm{O}_{3}: \mathrm{C} 71.97 ; \mathrm{H} 8.86$, found $\mathrm{C} 71.91 ; \mathrm{H} 9.02$.<smiles></smiles>

\section{2-Ethyl-3-trityloxymethyl-oxirane (12):}

A mixture of activated molecular sieves $3 \AA(4.0 \mathrm{~g}),(D)$-(-)-diethyl tartrate $(1.00 \mathrm{~mL}, 5.88$ mmol, $12 \mathrm{~mol} \%)$, Ti(Oi-Pr) $)_{4}(1.30 \mathrm{~mL}, 4.41 \mathrm{mmol}, 9 \mathrm{~mol} \%)$, and tert $-\mathrm{BuO}_{2} \mathrm{H}(17.8 \mathrm{~mL}$ of a $5.5 \mathrm{M}$ solution in decane, $98.02 \mathrm{mmol}$ ) in $80 \mathrm{~mL}$ of anhydrous dichloromethane was stirred at $-15^{\circ} \mathrm{C}$ under argon. After an aging period of $30 \mathrm{~min},(E)$-pent-2-en-1-ol (4.22 g, $\left.49.01 \mathrm{mmol}\right)$ was added dropwise over a 45 min period. After 5 days at $-15^{\circ} \mathrm{C}$, the excess of peroxide was destroyed by addition of $\mathrm{P}(\mathrm{OMe})_{3}(8.67 \mathrm{~mL}, 73.52 \mathrm{mmol})$. After $1.5 \mathrm{~h}$, the temperature was allowed to reach $\mathrm{rt}$ and when TLC plates showed that all the peroxide disappeared, $\mathrm{Et}_{3} \mathrm{~N}$ (20.56 mL, $147.03 \mathrm{mmol})$, DMAP (598 mg, $4.90 \mathrm{mmol})$ and trityl chloride $(27.33 \mathrm{~g}, 98.02$ mmol) were added. After $24 \mathrm{~h}$ of reaction, the mixture was filtered through a short celite pad, washed with a saturated aqueous $\mathrm{NaHCO}_{3}$ solution, the organic phase was dried over anhydrous $\mathrm{Na}_{2} \mathrm{SO}_{4}$ and the solvent was removed under vacuum. The residue was purified by flash chromatography (heptane/EtOAc, 30:1) affording $10.34 \mathrm{~g}$ (61\% over two steps) of the epoxide $\mathbf{1 2}$ as a colorless deliquescent solid.

$[\alpha]^{22}+4.3$ (c 3.37, $\mathrm{CH}_{2} \mathrm{Cl}_{2}$ ); $\mathrm{R} f 0.39$ (heptane/EtOAc, 10:1); IR (film) 3058, 3023, 2969, 2927, 2873, 1490, 1448, 1068, $706 \mathrm{~cm}^{-1}$; ${ }^{1} \mathrm{H}$ NMR $\left(300 \mathrm{MHz}, \mathrm{CDCl}_{3}\right) \delta_{\mathrm{H}} 0.98(\mathrm{t}, 3 \mathrm{H}, J=7.5$ $\mathrm{Hz}), 1.58(\mathrm{~m}, 2 \mathrm{H}), 2.81(\mathrm{td}, 1 \mathrm{H}, J=2.2,7.6 \mathrm{~Hz}), 2.94(\mathrm{~m}, 1 \mathrm{H}), 3.13(\mathrm{dd}, 1 \mathrm{H}, J=5.4,10.7$ $\mathrm{Hz}), 3.27(\mathrm{dd}, 1 \mathrm{H}, J=3.4,10.7 \mathrm{~Hz}), 7.27(\mathrm{~m}, 9 \mathrm{H}), 7.46(\mathrm{~m}, 6 \mathrm{H}) ;{ }^{13} \mathrm{C} \mathrm{NMR}\left(75 \mathrm{MHz}, \mathrm{CDCl}_{3}\right)$ $\delta_{\mathrm{C}} 9.8,24.8,56.9,57.4,64.9,86.6,127.0,127.7,128.7,143.9 ; \mathrm{MS}(\mathrm{TOF}) \mathrm{m} / \mathrm{z} 367.2([\mathrm{M}+$ $\mathrm{Na}]^{+}$); Anal. calcd for $\mathrm{C}_{24} \mathrm{H}_{24} \mathrm{O}_{2}$ : C 83.69; $\mathrm{H} 7.02$, found $\mathrm{C} 83.65 ; \mathrm{H} 7.02$. 
<smiles>C=C([C@H](CC)[C@@H](O)CO)[Si](C)(C)C</smiles>

\section{3-Ethyl-4-trimethylsilanyl-pent-4-ene-1,2-diol (14):}

Copper iodide (42 mg, $0.220 \mathrm{mmol})$ and $\mathrm{Me}_{2} \mathrm{~S}(40 \mu \mathrm{L})$ were mixed in anhydrous THF $(1 \mathrm{~mL})$ under argon. The flask was placed into an ice bath and a 1 M THF solution of 1trimethylsilylvinylmagnesium bromide $(1.30 \mathrm{~mL}, 1.30 \mathrm{mmol})$ was added slowly. After 15 min of stirring, a solution of epoxide $12(383 \mathrm{mg}, 1.11 \mathrm{mmol})$ in THF (1 mL) was added. After $48 \mathrm{~h}$ of reaction, the temperature was allowed to reach $\mathrm{rt}$ and $4 \mathrm{~h}$ later the reaction mixture was poured into a separatory funnel containing $25 \mathrm{~mL}$ of a saturated aqueous $\mathrm{NH}_{4} \mathrm{Cl}$ solution. After extraction with $\mathrm{CH}_{2} \mathrm{Cl}_{2}$, the organic phases were dried over anhydrous $\mathrm{Na}_{2} \mathrm{SO}_{4}$ and the solution was concentrated affording alcohol $\mathbf{1 3}$ as a colorless oil. The crude alcohol 13 was then taken in $\mathrm{MeOH}(11 \mathrm{~mL})$, PPTS $(28 \mathrm{mg}, 0.11 \mathrm{mmol})$ was added and the solution was refluxed during $1.5 \mathrm{~h}$ under argon. The reaction was quenched by addition of $300 \mathrm{mg}$ of $\mathrm{NaHCO}_{3}$, the solution was filtered and the solvent removed under reduced pressure. The residue was purified by flash chromatography (heptane/EtOAc, 2:1) affording $173 \mathrm{mg}$ of diol 14 (77\% over two steps) as a colorless oil.

$[\alpha]^{22}{ }_{\mathrm{D}}-4.87$ (c 0.91, $\mathrm{CH}_{2} \mathrm{Cl}_{2}$ ); $\mathrm{R} f 0.17$ (heptane/EtOAc, 1:1); IR (film) 3368, 3049, 2957, 2874, 1455, 1406, $1248 \mathrm{~cm}^{-1} ;{ }^{1} \mathrm{H}$ NMR $\left(300 \mathrm{MHz}, \mathrm{CDCl}_{3}\right) \delta_{\mathrm{H}} 0.10(\mathrm{~s}, 9 \mathrm{H}), 0.82(\mathrm{t}, 3 \mathrm{H}, J=$ $7.4 \mathrm{~Hz}), 1.49(\mathrm{~m}, 1 \mathrm{H}), 1.76(\mathrm{~m}, 1 \mathrm{H}), 1.95(\mathrm{brs}, 1 \mathrm{H}), 2.19(\mathrm{~m}, 2 \mathrm{H}, J=3.5 \mathrm{~Hz}), 3.41(\mathrm{~m}, 1 \mathrm{H}, J$ $=8.6,2.5 \mathrm{~Hz}), 3.62(\mathrm{~d}, 2 \mathrm{H}, J=8.2 \mathrm{~Hz}), 5.56(\mathrm{~d}, 1 \mathrm{H}, J=2.4 \mathrm{~Hz}), 5.66(\mathrm{~d}, 1 \mathrm{H}, J=2.3 \mathrm{~Hz})$; ${ }^{13} \mathrm{C}$ NMR $\left(75 \mathrm{MHz}, \mathrm{CDCl}_{3}\right) \delta_{\mathrm{C}}-0.7,11.9,23.3,50.0,65.9,74.8,127.0,152.6$. MS (TOF) $\mathrm{m} / \mathrm{z}$ $225\left([\mathrm{M}+\mathrm{Na}]^{+}\right)$; HRMS (TOF) $\mathrm{m} / \mathrm{z}$ calcd for $\mathrm{C}_{10} \mathrm{H}_{22} \mathrm{O}_{2} \mathrm{Na}[\mathrm{M}+\mathrm{Na}]^{+} 225.1287$, found 225.1295; Anal. calcd for $\mathrm{C}_{10} \mathrm{H}_{22} \mathrm{O}_{2} \mathrm{Si}$ : C 59.35; H 10.96, found C 59.02; $\mathrm{H} 10.99$.

\section{3-Ethyl-4-trimethylsilanyl-1-trityloxy-pent-4-en-2-ol (13):}

Alcohol 13 was purified by column chromatography (heptane/EtOAc, 30:1).

${ }^{1} \mathrm{H}$ NMR $\left(300 \mathrm{MHz}, \mathrm{CDCl}_{3}\right) \delta_{\mathrm{H}}-0.05(\mathrm{~s}, 3 \mathrm{H}), 0.77(\mathrm{t}, 3 \mathrm{H}, J=7.4 \mathrm{~Hz}), 1.40(\mathrm{~m}, 1 \mathrm{H}), 1.76(\mathrm{~m}$, $1 \mathrm{H}), 2.16(\mathrm{ddd}, 1 \mathrm{H}, J=3.3,8.3,10.2 \mathrm{~Hz}), 2.46(\mathrm{~d}, 1 \mathrm{H}, J=2.5 \mathrm{~Hz}), 2.95(\mathrm{t}, 1 \mathrm{H}, J=9.2 \mathrm{~Hz})$, $3.16(\mathrm{dd}, 1 \mathrm{H}, J=3.4,9.3 \mathrm{~Hz}), 3.79(\mathrm{~m}, 1 \mathrm{H}), 5.37(\mathrm{~d}, 1 \mathrm{H}, J=2.4 \mathrm{~Hz}), 5.53(\mathrm{~d}, 1 \mathrm{H}, J=2.3$ $\mathrm{Hz}), 7.27(\mathrm{~m}, 9 \mathrm{H}), 7.42(\mathrm{~m}, 6 \mathrm{H})$.<smiles>C=C[C@@H](CC)[C@H](O)CO</smiles>

\section{3-Ethyl-pent-4-ene-1,2-diol (15):}

To a slurry of $\mathrm{NaH}(60 \%$ dispersion in mineral oil, $2.03 \mathrm{~g}, 50.67 \mathrm{mmol})$ in HMPA $(40 \mathrm{~mL})$ was added diol 14 (4.661 g, $23.03 \mathrm{mmol})$ dissolved in anhydrous THF (40 mL). After $20 \mathrm{~h}$ of stirring at $25{ }^{\circ} \mathrm{C}$ under an argon atmosphere, the reaction mixture was poured in a saturated aqueous $\mathrm{NH}_{4} \mathrm{Cl}$ solution. After extraction with EtOAc, the organic extract was washed with water, dried over anhydrous $\mathrm{Na}_{2} \mathrm{SO}_{4}$ and the solvent was removed under vacuum leaving a residue that was purified by distillation $\left(75^{\circ} \mathrm{C}, 4.10^{-2} \mathrm{mmHg}\right)$ affording $2.69 \mathrm{~g} \mathrm{(89 \% )} \mathrm{of} \mathrm{diol}$ $\mathbf{1 5}$ as a colorless oil.

$[\alpha]^{23}{ }_{\mathrm{D}}+12.1$ (c 1.14, $\mathrm{CHCl}_{3}$ ); Rf 0.11 (heptane/EtOAc, 1:1); IR (film) 3369, 3077, 2963, 2929, 2874, 1639, 1420, $1071 \mathrm{~cm}^{-1} ;{ }^{1} \mathrm{H}$ NMR (300 MHz, $\left.\mathrm{CDCl}_{3}\right) \delta_{\mathrm{H}} 0.88(\mathrm{t}, 3 \mathrm{H}, J=7.4 \mathrm{~Hz})$, 
$1.26(\mathrm{~m}, 1 \mathrm{H}), 1.73(\mathrm{~m}, 1 \mathrm{H}), 2.05(\mathrm{~m}, 1 \mathrm{H}), 2.81$ (brs, $2 \mathrm{H}), 3.46(\mathrm{dd}, 1 \mathrm{H}, J=7.9,10.9 \mathrm{~Hz}), 3.56$ $(\mathrm{td}, 1 \mathrm{H}, J=2.6,7.7 \mathrm{~Hz}), 3.67(\mathrm{dd}, 1 \mathrm{H}, J=2.6,10.9 \mathrm{~Hz}), 5.09(\mathrm{~m}, 2 \mathrm{H}), 5.52(\mathrm{td}, 1 \mathrm{H}, J=9.8$, $16.9 \mathrm{~Hz}) ;{ }^{13} \mathrm{C} \mathrm{NMR}\left(75 \mathrm{MHz}, \mathrm{CDCl}_{3}\right) \delta_{\mathrm{C}} 11.6,23.4,49.9,65.2,74.4,117.6,138.2$; MS (TOF) $\mathrm{m} / \mathrm{z} 153.1\left([\mathrm{M}+\mathrm{Na}]^{+}\right)$; HRMS (TOF) $\mathrm{m} / \mathrm{z}$ calcd for $\mathrm{C}_{7} \mathrm{H}_{14} \mathrm{O}_{2} \mathrm{Na}[\mathrm{M}+\mathrm{Na}]^{+} 153.0891$, found 153.0897 .<smiles>C=CC(CC)C(=O)O</smiles>

\section{2-Ethyl-but-3-enoic acid (7):}

A solution of $\mathrm{NaIO}_{4}(2.10 \mathrm{~g}, 97 \mathrm{mmol})$ in $30 \mathrm{~mL}$ of water was mixed with an ether solution $(30 \mathrm{~mL})$ of diol 15 (2.097 g, $16.108 \mathrm{mmol})$. After $24 \mathrm{~h}$ of stirring, the organic phase was separated and the aqueous phase was extracted with $3 \times 20 \mathrm{~mL}$ of ether. To this ether solution was added 2-methyl-2-butene (34 mL), a solution of $\mathrm{NaClO}_{2}(7.28 \mathrm{~g}, 80.54 \mathrm{mmol})$ in water $(30 \mathrm{~mL})$ and $\mathrm{NaH}_{2} \mathrm{PO}_{4}\left(\mathrm{H}_{2} \mathrm{O}\right)_{2}(12.56 \mathrm{~g}, 80.54 \mathrm{mmol})$. The biphasic mixture was vigorously stirred during $3 \mathrm{~h}$. The reaction mixture was made acidic with addition of a $1 \mathrm{M} \mathrm{HCl}$ aqueous solution $(25 \mathrm{~mL})$, the organic phase was separated, and the aqueous phase was extracted with $50 \mathrm{~mL}$ of ether. The solvent was concentrated under a controlled vacuum to about $50 \mathrm{~mL}$. This solution was extracted with $2 \times 25 \mathrm{~mL}$ of a saturated aqueous $\mathrm{NaHCO}_{3}$ solution and after separation, the aqueous phase containing acid 7 sodium salt was made acidic with addition of $2 \mathrm{M} \mathrm{HCl}$ and extracted with ether. After drying over anhydrous $\mathrm{Na}_{2} \mathrm{SO}_{4}$ and removal of the solvent under vacuum, the residue was purified by distillation affording $1.304 \mathrm{~g} \mathrm{(71 \% )}$ of the acid 7.

$[\alpha]^{23}{ }_{\mathrm{D}}-47.9$ (c 1.62, $\mathrm{CHCl}_{3}$ ); IR (film) 2967, 2935, 2878, 1702, 1639, $1216 \mathrm{~cm}^{-1} ;{ }^{1} \mathrm{H}$ NMR $\left(300 \mathrm{MHz}, \mathrm{CDCl}_{3}\right) \delta_{\mathrm{H}} 0.94(\mathrm{t}, 3 \mathrm{H}, J=7.5 \mathrm{~Hz}), 1.61(\mathrm{~m}, 1 \mathrm{H}, J=7.4 \mathrm{~Hz}), 1.83(\mathrm{~m}, 1 \mathrm{H}, J=6.6$ $\mathrm{Hz}), 2.96(\mathrm{q}, 1 \mathrm{H}, J=7.6,7.9 \mathrm{~Hz}), 5.15(\mathrm{~s}, 1 \mathrm{H}), 5.21(\mathrm{~d}, 1 \mathrm{H}, J=3.9 \mathrm{~Hz}), 5.82(\mathrm{~m}, 1 \mathrm{H}, J=$ $8.8 \mathrm{~Hz}) ;{ }^{13} \mathrm{C} \mathrm{NMR}\left(75 \mathrm{MHz}, \mathrm{CDCl}_{3}\right) \delta_{\mathrm{C}} 11.6,25.4,51.8,117.9,135.4,180.7$; Anal. calcd for $\mathrm{C}_{6} \mathrm{H}_{10} \mathrm{O}_{2}$ : C 63.14; $\mathrm{H} 8.83$, found $\mathrm{C} 60.19 ; \mathrm{H} 8.82$.

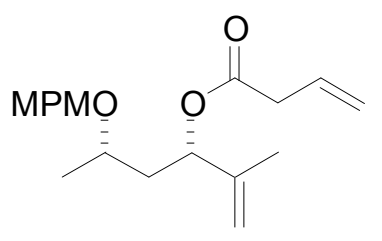

\section{But-3-enoic acid 1-[2-(4-methoxy-benzyloxy)-propyl]-2-methyl-allyl ester (16):}

Alcohol 6 (989 mg, $3.95 \mathrm{mmol})$ was dissolved in $\mathrm{Et}_{3} \mathrm{~N}(1.66 \mathrm{~mL}, 11.85 \mathrm{mmol})$ at $0{ }^{\circ} \mathrm{C}$ under argon and crotonoyl chloride $(640 \mu \mathrm{L}, 6.72 \mathrm{mmol})$ was added dropwise. The thick reaction mixture was stirred during $50 \mathrm{~min}$ and a $3 \mathrm{M}$ hydrochloric acid $(2 \mathrm{~mL})$ was added at $0{ }^{\circ} \mathrm{C}$. The mixture was then poured into a separatory funnel containing $50 \mathrm{~mL}$ of a saturated aqueous $\mathrm{NaHCO}_{3}$ solution. After extraction with EtOAc and drying over anhydrous $\mathrm{Na}_{2} \mathrm{SO}_{4}$, the solvent was removed under vacuum. The residue was purified by flash chromatography (heptane/EtOAc, 20:1) affording $1.07 \mathrm{~g}(85 \%)$ of ester 16 as a colorless oil.

$[\alpha]_{\mathrm{D}}^{23}+13.7$ (c 0.90, $\mathrm{CHCl}_{3}$ ); $\mathrm{R} f 0.39$ (heptane/EtOAc, 2:1); IR (film) 2969, 1732, 1512, $1245,1171 \mathrm{~cm}^{-1} ;{ }^{1} \mathrm{H}$ NMR $\left(300 \mathrm{MHz}, \mathrm{CDCl}_{3}\right) \delta_{\mathrm{H}} 1.22(\mathrm{~d}, 3 \mathrm{H}, J=6.1 \mathrm{~Hz}), 1.68(\mathrm{~m}, 1 \mathrm{H}), 1.70$ $(\mathrm{s}, 3 \mathrm{H}), 2.04(\mathrm{~m}, 1 \mathrm{H}), 3.04(\mathrm{td}, 2 \mathrm{H}, J=1.3,6.9 \mathrm{~Hz}), 3.51(\mathrm{~m}, 1 \mathrm{H}), 3.80(\mathrm{~s}, 3 \mathrm{H}), 4.33(\mathrm{~d}, 1 \mathrm{H}, J$ $=11.1 \mathrm{~Hz}), 4.48(\mathrm{~d}, 1 \mathrm{H}, J=11.1 \mathrm{~Hz}), 4.88(\mathrm{t}, 1 \mathrm{H}, J=1.5 \mathrm{~Hz}), 4.94(\mathrm{~m}, 1 \mathrm{H}), 5.11(\mathrm{~m}, 1 \mathrm{H})$, $5.16(\mathrm{~m}, 1 \mathrm{H}), 5.35(\mathrm{t}, 1 \mathrm{H}, J=7.0 \mathrm{~Hz}), 5.82-5.96(\mathrm{~m}, 1 \mathrm{H}), 6.87(\mathrm{~d}, 2 \mathrm{H}, J=8.7 \mathrm{~Hz}), 7.26(\mathrm{~d}$, 
$2 \mathrm{H}, J=8.6 \mathrm{H}) ;{ }^{13} \mathrm{C}$ NMR $\left(75 \mathrm{MHz}, \mathrm{CDCl}_{3}\right) \delta_{\mathrm{C}} 18.1,19.8,39.5,40.1,55.4,70.1,71.7,75.4$, $113.4,113.9,118.6,129.4,130.4,130.9,143.1,159.2,170.7$; MS (TOF) m/z 341.1 ([M + $\mathrm{Na}]^{+}$); HRMS (TOF) $\mathrm{m} / \mathrm{z}$ calcd for $\mathrm{C}_{19} \mathrm{H}_{26} \mathrm{O}_{4} \mathrm{Na}[\mathrm{M}+\mathrm{Na}]^{+} 341.1729$, found 341.1744; Anal. calcd for $\mathrm{C}_{19} \mathrm{H}_{26} \mathrm{O}_{4}$ : C 71.67; $\mathrm{H} 8.23$, found $\mathrm{C} 71.25 ; \mathrm{H}$ 8.24.

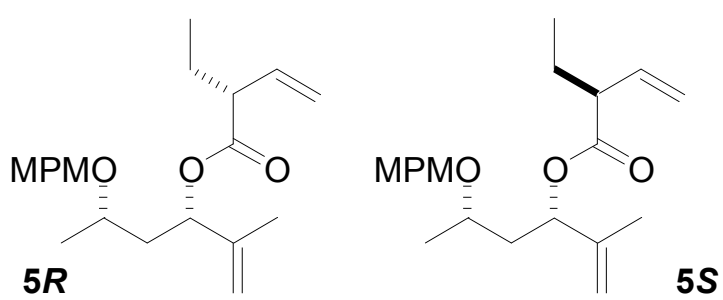

2-Ethyl-but-3-enoic acid 1-[2-(4-methoxy-benzyloxy)-propyl]-2-methyl-allyl ester (5R, 5S):

By coupling with acid 7:

Allylic alcohol 6 (657 mg, $2.63 \mathrm{mmol})$, acid 7 (270 mg, $2.36 \mathrm{mmol})$, DMAP (96 mg, 0.79 $\mathrm{mmol}$ ) and DCC (650 mg, $3.15 \mathrm{mmol}$ ) were dissolved in $10 \mathrm{~mL}$ of dry $\mathrm{CH}_{2} \mathrm{Cl}_{2}$ under argon at rt. After $5 \mathrm{~h}$ of stirring, the reaction mixture was filtered. The solvent was removed under vacuum and the residue was purified by column chromatography (heptane/EtOAc, 20:1) affording esters $\mathbf{5} \boldsymbol{R}$ and $\mathbf{5 S}$ (471 $\mathrm{mg}, \mathbf{5 7} \%$, colorless oil) as an inseparable mixture of diastereomers (5R/5S: 6/4 evaluated by ${ }^{1} \mathrm{H}$ NMR).

$[\alpha]_{\mathrm{D}}^{22}+0.11$ (c 1.03, $\mathrm{CHCl}_{3}$ ); Rf 0.50 (heptane/EtOAc, 2:1); IR (film) 2966, 2874, 1730, $1615,1512,1245 \mathrm{~cm}^{-1} ;{ }^{1} \mathrm{H}$ NMR $\left(300 \mathrm{MHz}, \mathrm{CDCl}_{3}\right)$ for ester $\mathbf{5} \boldsymbol{R} \delta_{\mathrm{H}} 0.88(\mathrm{t}, 3 \mathrm{H}, J=7.4 \mathrm{~Hz})$, $1.21(\mathrm{~d}, 3 \mathrm{H}, J=6.1 \mathrm{~Hz}), 1.54(\mathrm{~m}, 1 \mathrm{H}, J=7.4 \mathrm{~Hz}), 1.64-1.78(\mathrm{~m}, 5 \mathrm{H}), 2.05(\mathrm{~m}, 1 \mathrm{H}), 2.87(\mathrm{~m}$, $1 \mathrm{H}), 3.48(\mathrm{~m}, 1 \mathrm{H}), 3.79(\mathrm{~s}, 3 \mathrm{H}), 4.36(\mathrm{~d}, 1 \mathrm{H}, J=11.1 \mathrm{~Hz}), 4.46(\mathrm{~d}, 1 \mathrm{H}, J=11.1 \mathrm{~Hz}), 4.88(\mathrm{~m}$, $1 \mathrm{H}), 4.94(\mathrm{~m}, 1 \mathrm{H}), 5.09(\mathrm{~d}, 1 \mathrm{H}, J=4.4 \mathrm{~Hz}), 5.12(\mathrm{~d}, 1 \mathrm{H}, J=5.0 \mathrm{~Hz}), 5.31(\mathrm{t}, 1 \mathrm{H}, J=8.2 \mathrm{~Hz})$, $5.77(\mathrm{~m}, 1 \mathrm{H}), 6.88(\mathrm{~d}, 2 \mathrm{H}, J=8.6 \mathrm{~Hz}), 7.26(\mathrm{~d}, 2 \mathrm{H}, J=8.3 \mathrm{~Hz}) ;{ }^{13} \mathrm{C} \mathrm{NMR}\left(75 \mathrm{MHz}, \mathrm{CDCl}_{3}\right)$ $\delta_{\mathrm{C}} 11.7,18.0,19.7,25.4,40.1,52.3,55.4,70.1,71.5,75.0,113.3,113.9,117.3,129.3,130.9$, 136.1, 143.1, 159.2, 173.1; MS (TOF) m/z $369.2\left([\mathrm{M}+\mathrm{Na}]^{+}\right)$; HRMS (TOF) $\mathrm{m} / \mathrm{z}$ calcd for $\mathrm{C}_{21} \mathrm{H}_{30} \mathrm{O}_{4} \mathrm{Na}[\mathrm{M}+\mathrm{Na}]^{+}$369.2042, found 369.2033; Anal. calcd for $\mathrm{C}_{21} \mathrm{H}_{30} \mathrm{O}_{4}: \mathrm{C} 72.80 ; \mathrm{H}$ 8.73 , foundC $72.82 ; \mathrm{H} 8.76$.

By alkylation of ester 16:

To a solution of $i$ - $\mathrm{Pr}_{2} \mathrm{NH}(510 \mu \mathrm{L}, 3.66 \mathrm{mmol})$ in anhydrous $\mathrm{THF}(15 \mathrm{~mL})$ at $0{ }^{\circ} \mathrm{C}$ under argon was added $n$-BuLi $(2.25 \mathrm{~mL}$ of a $1.6 \mathrm{M}$ hexane solution, $3.59 \mathrm{mmol})$. This solution was stirred at $\mathrm{rt}$ during $30 \mathrm{~min}$ before being cooled at $-78^{\circ} \mathrm{C}$. A solution of the ester $16(1.06 \mathrm{~g}$, $3.33 \mathrm{mmol})$ in THF $(4 \mathrm{~mL})$ was introduced followed by HMPA $(1.74 \mathrm{~mL}, 10.0 \mathrm{mmol})$. After $10 \mathrm{~min}$ of stirring, ethyl iodide $(290 \mu \mathrm{L}, 3.66 \mathrm{mmol})$ was added and the reaction was quenched $20 \mathrm{~min}$ later by addition of $\mathrm{AcOH}(250 \mu \mathrm{L})$ in THF $(1 \mathrm{~mL})$. The reaction mixture was allowed to reach $\mathrm{rt}$ and poured into a separatory funnel containing $150 \mathrm{~mL}$ of a saturated aqueous $\mathrm{NH}_{4} \mathrm{Cl}$ solution. After extraction with $3 \times 50 \mathrm{~mL}$ of EtOAc, the organic phase was dried over anhydrous $\mathrm{Na}_{2} \mathrm{SO}_{4}$ and the solvent was removed under vacuum. The residue was purified by column chromatography (heptane/EtOAc, 20:1) affording esters $\mathbf{5} \boldsymbol{R}$ and $\mathbf{5} \boldsymbol{S}$ (901 $\mathrm{mg}, 78 \%$, colorless oil) as an inseparable mixture of diastereomers $(\mathbf{5 R} / \mathbf{5 S}: 4 / 6$ as evaluated by ${ }^{1} \mathrm{H}$ NMR).

By racemization of recovered ester $5 S$ :

To a solution of $i$ - $\mathrm{Pr}_{2} \mathrm{NH}(190 \mu \mathrm{L}, 1.36 \mathrm{mmol})$ in anhydrous $\mathrm{THF}(10 \mathrm{~mL})$ at $0{ }^{\circ} \mathrm{C}$ under argon was added $n$-BuLi ( $840 \mu \mathrm{L}$ of a $1.6 \mathrm{M}$ hexane solution, $1.34 \mathrm{mmol}$ ). The solution was stirred 
at $\mathrm{rt}$ during $30 \mathrm{~min}$ before being cooled at $-78{ }^{\circ} \mathrm{C}$. Then a solution of the ester $\mathbf{5 S}(430 \mathrm{mg}$, $1.24 \mathrm{mmol})$ in THF (2 mL) was introduced. After $10 \mathrm{~min}$ of stirring the reaction was quenched by addition of $\mathrm{AcOH}(180 \mu \mathrm{L}$ diluted with $0.5 \mathrm{~mL}$ of THF). The reaction mixture was allowed to reach $\mathrm{rt}$ and poured into a separatory funnel containing $50 \mathrm{~mL}$ of a saturated aqueous $\mathrm{NH}_{4} \mathrm{Cl}$ solution. After extraction with $3 \times 10 \mathrm{~mL}$ of EtOAc, the organic phase was dried over anhydrous $\mathrm{Na}_{2} \mathrm{SO}_{4}$ and the solvent was removed under vacuum. The residue was purified by column chromatography (heptane/EtOAc, 20:1) affording esters $\mathbf{5} \boldsymbol{R}$ and $\mathbf{5} \boldsymbol{S}$ (430 $\mathrm{mg}, 100 \%$, colorless oil) as a mixture of inseparable diastereomers $(\mathbf{5} \boldsymbol{R} / \mathbf{5 S}: 1 / 1$ evaluated by ${ }^{1} \mathrm{H}$ NMR).<smiles>CC[C@H]1C=C(C)[C@@H](CC(C)O[Na])OC1=O</smiles>

\section{3-Ethyl-6-[2-(4-methoxy-benzyloxy)-propyl]-5-methyl-3,6-dihydro-pyran-2-one (4):}

A mixture of esters $\mathbf{5} \boldsymbol{R} / \mathbf{5} \mathrm{S}$ (ratio: $6 / 4,104 \mathrm{mg}, 0.300 \mathrm{mmol}$ ) was dissolved in $\mathrm{CH}_{2} \mathrm{Cl}_{2}(15 \mathrm{~mL}$ ) under argon, then second-generation Grubbs' catalyst (15 mg, $0.018 \mathrm{mmol})$ was introduced and the reaction mixture was refluxed during 3 days. The solvent was removed under vacuum and the residue was purified by flash chromatography (heptane/EtOAc, 5:1) affording $71 \mathrm{mg}$ (75\%) of lactone 4 as a colorless oil.

$[\alpha]_{\mathrm{D}}^{22}+8.42\left(c\right.$ 0.94, $\left.\mathrm{CHCl}_{3}\right)$; Rf 0.28 (heptane/EtOAc, 2:1); IR (film) 3469, 2967, 2931, 1736, 1611, 1513, $1249 \mathrm{~cm}^{-1} ;{ }^{1} \mathrm{H}$ NMR $\left(500 \mathrm{MHz}, \mathrm{CDCl}_{3}\right) \delta_{\mathrm{H}} 0.98(\mathrm{t}, 3 \mathrm{H}, J=7.4 \mathrm{~Hz}), 1.28$ $(\mathrm{d}, 3 \mathrm{H}, J=6.1 \mathrm{~Hz}), 1.75(\mathrm{~s}, 3 \mathrm{H}), 17.2-1.85(\mathrm{~m}, 2 \mathrm{H}), 1.87$ (ddd, $1 \mathrm{H}, J=3.1,8.3,14.3 \mathrm{~Hz})$; 2.00 (ddd, 1H, $J=4.5,9.9,14.3 \mathrm{~Hz}), 2.96(\mathrm{~m}, 1 \mathrm{H}), 3.80(\mathrm{~s}, 3 \mathrm{H}), 3.86(\mathrm{~m}, 1 \mathrm{H}), 4.38(\mathrm{~d}, 1 \mathrm{H}, J$ $=11.1 \mathrm{~Hz}), 4.50(\mathrm{~d}, 1 \mathrm{H}, J=11.1 \mathrm{~Hz}), 4.79(\mathrm{dt}, 1 \mathrm{H}, J=3.1,9.9 \mathrm{~Hz}), 5.46(\mathrm{~m}, 1 \mathrm{H}), 6.87(\mathrm{dt}$, $2 \mathrm{H}, J=8.6,1.9 \mathrm{~Hz}), 7.25(\mathrm{dt}, 2 \mathrm{H}, J=2.1,8.7 \mathrm{~Hz}) ;{ }^{13} \mathrm{C} \mathrm{NMR}\left(75 \mathrm{MHz}, \mathrm{CDCl}_{3}\right) \delta_{\mathrm{C}} 11.3,18.9$, 19.3, 26.9, 41.1, 42.2, 55.4, 70.4, 71.5, 79.9, 113.9, 121.2, 129.1, 130.9, 133.2, 159.3, 172.1; MS (TOF) $\mathrm{m} / \mathrm{z} 341.2\left([\mathrm{M}+\mathrm{Na}]^{+}\right)$; HRMS (TOF) $\mathrm{m} / \mathrm{z}$ calcd for $\mathrm{C}_{19} \mathrm{H}_{26} \mathrm{O}_{4} \mathrm{Na}[\mathrm{M}+\mathrm{Na}]^{+}$ 341.1729, found 341.1752; Anal. calcd for $\mathrm{C}_{19} \mathrm{H}_{26} \mathrm{O}_{4}$ : C 71.67; $\mathrm{H} \mathrm{8.23}$, found $\mathrm{C} 71.32 ; \mathrm{H}$ 8.19 .

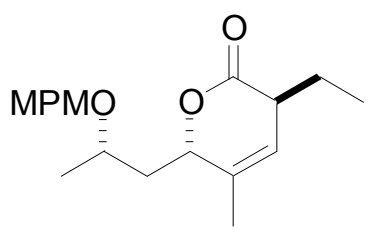

\section{Lactone (4a):}

$[\alpha]_{\mathrm{D}}^{23}+67.5$ (c 0.70, $\mathrm{CHCl}_{3}$ ); Rf 0.29 (heptane/EtOAc, 2:1); IR (film) 2966, 1732, 1612, $1513,1247 \mathrm{~cm}^{-1} ;{ }^{1} \mathrm{H}$ NMR $\left(500 \mathrm{MHz}, \mathrm{CDCl}_{3}\right) \delta_{\mathrm{H}} 0.93(\mathrm{t}, 3 \mathrm{H}, J=7.5 \mathrm{~Hz}), 1.28(\mathrm{~d}, 3 \mathrm{H}, J=6.1$ $\mathrm{Hz}), 1.76(\mathrm{~s}, 3 \mathrm{H}), 1.82(\mathrm{~m}, 3 \mathrm{H}), 2.02(\mathrm{ddd}, 1 \mathrm{H}, J=5.1,9.1,14.3 \mathrm{~Hz}), 2.92(\mathrm{~m}, 1 \mathrm{H}), 3.80(\mathrm{~m}$, $4 \mathrm{H}), 4.35$ (d, 1H, $J=11.0 \mathrm{~Hz}), 4.49(\mathrm{~d}, 1 \mathrm{H}, J=11.0 \mathrm{~Hz}), 4.78(\mathrm{dt}, 1 \mathrm{H}, J=2.9,8.9 \mathrm{~Hz}), 5.37$ $(\mathrm{s}, 1 \mathrm{H}), 6.87(\mathrm{~d}, 2 \mathrm{H}, J=8.4 \mathrm{~Hz}), 7.24(\mathrm{~d}, 2 \mathrm{H}, J=8.5 \mathrm{~Hz}) ;{ }^{13} \mathrm{C} \mathrm{NMR}\left(75 \mathrm{MHz}, \mathrm{CDCl}_{3}\right) \delta_{\mathrm{C}} 10.5$, 19.1, 19.5, 25.3, 40.2, 41.1, 55.4, 70.5, 71.4, 80.4, 113.9, 120.8, 129.5, 130.8, 133.9, 159.3, 172.2; MS (TOF) $\mathrm{m} / \mathrm{z} 341.2\left([\mathrm{M}+\mathrm{Na}]^{+}\right)$; HRMS (TOF) $\mathrm{m} / \mathrm{z}$ calcd for $\mathrm{C}_{19} \mathrm{H}_{26} \mathrm{O}_{4} \mathrm{Na}[\mathrm{M}+\mathrm{Na}]^{+}$ 341.1729 , found 341.1752 . 
<smiles>CCC1C=C(C)C(CC(C)O[Na])OC1=O</smiles>

Lactone (4b):

$[\alpha]_{\mathrm{D}}^{23}+109.2\left(c\right.$ 0.95, $\left.\mathrm{CHCl}_{3}\right)$; Rf 0.30 (heptane/EtOAc, 2:1); IR (film) 2965, 2931, 2874, $1731,1612,1512,1245 \mathrm{~cm}^{-1} ;{ }^{1} \mathrm{H}$ NMR $\left(500 \mathrm{MHz}, \mathrm{CDCl}_{3}\right) \delta_{\mathrm{H}} 0.98(\mathrm{t}, 3 \mathrm{H}, J=7.4 \mathrm{~Hz}), 1.22(\mathrm{~d}$, $3 \mathrm{H}, J=6.1 \mathrm{~Hz}), 1.56(\mathrm{~m}, 1 \mathrm{H}), 1.74(\mathrm{~s}, 3 \mathrm{H}), 1.79(\mathrm{~m}, 2 \mathrm{H}), 1.96(\mathrm{ddd}, 1 \mathrm{H}, J=1.7,10.5,14.5$ $\mathrm{Hz}), 2.93(\mathrm{~s}, 1 \mathrm{H}), 3.80(\mathrm{~s}, 3 \mathrm{H}), 3.97(\mathrm{~m}, 1 \mathrm{H}), 4.42(\mathrm{~d}, 1 \mathrm{H}, J=10.9 \mathrm{~Hz}), 4.58(\mathrm{~d}, 1 \mathrm{H}, J=11.0$ $\mathrm{Hz}), 4.98(\mathrm{~d}, 1 \mathrm{H}, J=11.0 \mathrm{~Hz}), 5.45(\mathrm{~s}, 1 \mathrm{H}), 6.87(\mathrm{~d}, 2 \mathrm{H}, J=8.5 \mathrm{~Hz}),), 7.28(\mathrm{~d}, 2 \mathrm{H}, J=8.5$ $\mathrm{Hz}) ;{ }^{13} \mathrm{C}$ NMR $\left(75 \mathrm{MHz}, \mathrm{CDCl}_{3}\right) \delta_{\mathrm{C}} 11.3,18.8,20.3,26.6,41.4,43.4,55.4,71.2,79.0,114.0$, 121.3, 129.5, 131.1, 133.8, 159.3, 172.4; MS (TOF) $\mathrm{m} / \mathrm{z} 341.2\left([\mathrm{M}+\mathrm{Na}]^{+}\right)$; HRMS (TOF) $\mathrm{m} / \mathrm{z}$ calcd for $\mathrm{C}_{19} \mathrm{H}_{26} \mathrm{O}_{4} \mathrm{Na}[\mathrm{M}+\mathrm{Na}]^{+} 341.1729$, found 341.1752 .<smiles>CC[C@H](/C=C(/C)[C@H](O)C[C@H](C)O[Na])CO</smiles>

\section{2-Ethyl-7-(4-methoxy-benzyloxy)-4-methyl-oct-3-ene-1,5-diol (17):}

Lactone $4(477 \mathrm{mg}, 1.50 \mathrm{mmol})$ was dissolved in anhydrous THF $(20 \mathrm{~mL})$ and the solution was cooled to $-78^{\circ} \mathrm{C}$. $\mathrm{LiAlH}_{4}(85.3 \mathrm{mg}, 2.25 \mathrm{mmol})$ was added, the flask was removed from the cold bath and allowed to reach rt. After $1 \mathrm{~h}$ of stirring, the reaction mixture was carefully poured into a separatory funnel containing $100 \mathrm{~mL}$ of a saturated aqueous $\mathrm{NH}_{4} \mathrm{Cl}$ solution. After extraction with EtOAc, and drying over anhydrous $\mathrm{Na}_{2} \mathrm{SO}_{4}$, the solvent was removed under reduced pressure. The residue was purified by flash chromatography (heptane/EtOAc, 1:1) affording $443 \mathrm{mg}(92 \%)$ of diol 17 as a colorless oil.

$[\alpha]^{23}{ }_{\mathrm{D}}+74.5\left(c\right.$ 1.44, $\mathrm{CHCl}_{3}$ ); Rf 0.26 (heptane/EtOAc, 1:2); IR (film) 3366, 2959, 2926, $2870,1612,1513,1245,1032 \mathrm{~cm}^{-1} ;{ }^{1} \mathrm{H}$ NMR $\left(300 \mathrm{MHz}, \mathrm{CDCl}_{3}\right) \delta_{\mathrm{H}} 0.83(\mathrm{t}, 3 \mathrm{H}, J=7.4 \mathrm{~Hz})$, $1.17(\mathrm{~m}, 1 \mathrm{H}), 1.26(\mathrm{~d}, 3 \mathrm{H}, J=6.0 \mathrm{~Hz}), 1.35(\mathrm{~m}, 1 \mathrm{H}), 1.52(\mathrm{dt}, 1 \mathrm{H}, J=3.2,14.3 \mathrm{~Hz}), 1.75(\mathrm{~d}$, $3 \mathrm{H}, J=1.2 \mathrm{~Hz}), 1.96(\mathrm{dt}, 1 \mathrm{H}, J=9.5,14.5 \mathrm{~Hz}), 2.51(\mathrm{~m}, 1 \mathrm{H}), 3.24(\mathrm{t}, 1 \mathrm{H}, J=10.0 \mathrm{~Hz}), 3.56$ $(\mathrm{dd}, 1 \mathrm{H}, J=10.3,4.4 \mathrm{~Hz}), 3.80(\mathrm{~m}, 4 \mathrm{H}), 4.39(\mathrm{~d}, 1 \mathrm{H}, J=10.8 \mathrm{~Hz}), 4.57(\mathrm{~d}, 1 \mathrm{H}, J=10.8 \mathrm{~Hz})$, $4.66(\mathrm{dd}, 1 \mathrm{H}, J=2.6,9.7 \mathrm{~Hz}), 4.96(\mathrm{~d}, 1 \mathrm{H}, J=9.8 \mathrm{~Hz}), 6.87(\mathrm{~d}, 2 \mathrm{H}, J=8.6 \mathrm{~Hz}), 7.26(\mathrm{~d}, 2 \mathrm{H}$, $J=8.6 \mathrm{~Hz}) ;{ }^{13} \mathrm{C}$ NMR $\left(75 \mathrm{MHz}, \mathrm{CDCl}_{3}\right) \delta_{\mathrm{C}} 12.0,18.5,19.9,24.9,41.7,42.2,55.4,66.1,69.2$, $70.3,75.7,114.1,129.6,130.3,140.3,159.4 ; \mathrm{MS}(\mathrm{TOF}) \mathrm{m} / \mathrm{z} 345.2\left([\mathrm{M}+\mathrm{Na}]^{+}\right)$; HRMS (TOF) $\mathrm{m} / \mathrm{z}$ calcd for $\mathrm{C}_{19} \mathrm{H}_{30} \mathrm{O}_{4} \mathrm{Na}[\mathrm{M}+\mathrm{Na}]^{+} 345.2042$, found 345.2053. 


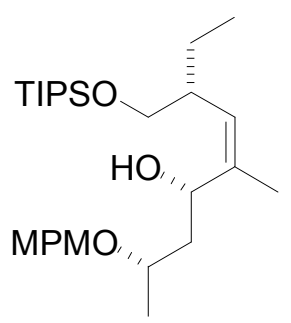

2-(4-Methoxy-benzyloxy)-5-methyl-7-triisopropylsilanyloxymethyl-non-5-en-4-ol (18):

Diol $17(647 \mathrm{mg}, 2.01 \mathrm{mmol})$ and triisopropylsilyl chloride $(670 \mu \mathrm{L}, 3.15 \mathrm{mmol})$ in dry pyridine $(4 \mathrm{~mL})$ were heated at $55{ }^{\circ} \mathrm{C}$ during 3 days under argon. The reaction mixture was poured into a separatory funnel containing $50 \mathrm{~mL}$ of a saturated aqueous $\mathrm{NH}_{4} \mathrm{Cl}$ solution. After extraction with $\mathrm{CH}_{2} \mathrm{Cl}_{2}$, the organic phase was dried over anhydrous $\mathrm{Na}_{2} \mathrm{SO}_{4}$ and the solvent was removed under vacuum. The residue was purified by flash chromatography (heptane/EtOAc, 10:1) affording $1.022 \mathrm{~g}(100 \%)$ of alcohol 18 as a colorless oil.

$[\alpha]_{\mathrm{D}}^{23}+18.4\left(c\right.$ 1.24, $\mathrm{CHCl}_{3}$ ); Rf 0.21 (heptane/EtOAc, 5:1).; IR (film) 3467, 2940, 2863, $1613,1513,1247 \mathrm{~cm}^{-1} ;{ }^{1} \mathrm{H}$ NMR $\left(300 \mathrm{MHz}, \mathrm{CDCl}_{3}\right) \delta_{\mathrm{H}} 0.81(\mathrm{t}, 3 \mathrm{H}, J=7.4 \mathrm{~Hz}), 0.97-1.18(\mathrm{~m}$, $22 \mathrm{H}), 1.25(\mathrm{~d}, 3 \mathrm{H}, J=6.1 \mathrm{~Hz}), 1.46-1.63(\mathrm{~m}, 2 \mathrm{H}), 1.72(\mathrm{~d}, 3 \mathrm{H}, J=1.3 \mathrm{~Hz}), 1.98(\mathrm{dt}, 1 \mathrm{H}, J=$ 8.9, $14.3 \mathrm{~Hz}), 2.49$ (m, 1H), 3.20 (brs, 1H), 3.52 (ddd, $2 \mathrm{H}, J=6.4,7.2,13.6 \mathrm{~Hz}), 3.76$ (m, $1 \mathrm{H}), 3.79(\mathrm{~s}, 3 \mathrm{H}), 4.36(\mathrm{~d}, 1 \mathrm{H}, J=10.9 \mathrm{~Hz}), 4.54(\mathrm{~d}, 1 \mathrm{H}, J=10.9 \mathrm{~Hz}), 4.70(\mathrm{dd}, 1 \mathrm{H}, J=3.5$, $9.2 \mathrm{~Hz}), 4.96(\mathrm{~d}, 1 \mathrm{H}, J=9.4 \mathrm{~Hz}), 6.87(\mathrm{dt}, 2 \mathrm{H}, J=2.1,8.7 \mathrm{~Hz}), 7.27(\mathrm{dt}, 2 \mathrm{H}, J=1.9,8.6 \mathrm{~Hz})$; 13C NMR (75 MHz, $\left.\mathrm{CDCl}_{3}\right) \delta_{\mathrm{C}} 12.1,12.2,12.5,17.9,18.2,18.3,20.0,24.8,42.0,42.1,55.4$, $67.1,68.8,70.3,75.2,114.0,129.5,129.7,130.7,139.2,159.3$; MS (TOF) m/z 501.3 ([M + $\mathrm{Na}]^{+}$); HRMS (TOF) $\mathrm{m} / \mathrm{z}$ calcd for $\mathrm{C}_{28} \mathrm{H}_{50} \mathrm{O}_{4} \mathrm{NaSi}[\mathrm{M}+\mathrm{Na}]^{+}$501.3376, found 501.3360;

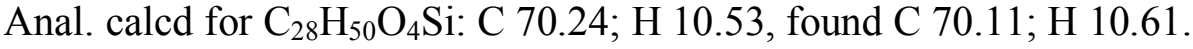

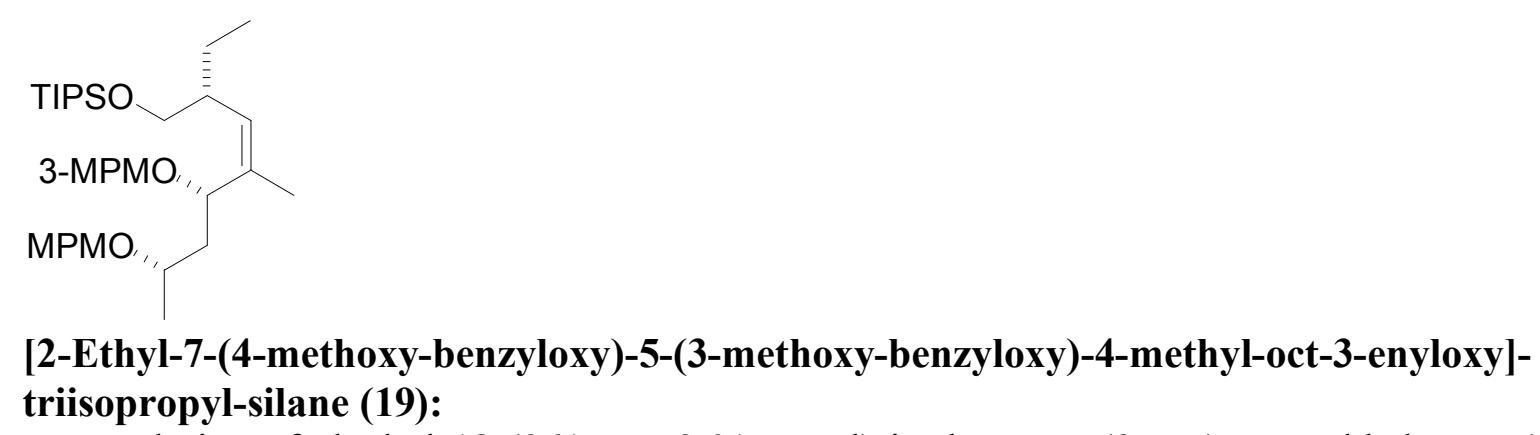

To a solution of alcohol $18(961 \mathrm{mg}, 2.01 \mathrm{mmol})$ in dry THF $(8 \mathrm{~mL})$ was added $\mathrm{NaH}(241$ $\mathrm{mg}, 60 \%$ in mineral oil, $6.02 \mathrm{mmol}$ ) followed by imidazole $(7 \mathrm{mg}, 0.10 \mathrm{mmol})$. After $15 \mathrm{~min}$ of stirring, 3-methoxybenzyl chloride was added along with tetrabutylammonium iodide (111 $\mathrm{mg}, 0.30 \mathrm{mmol}$ ). The reaction mixture was refluxed during $1.5 \mathrm{~h}$ under argon and then carefully poured into a separatory funnel containing $100 \mathrm{~mL}$ of a saturated aqueous $\mathrm{NH}_{4} \mathrm{Cl}$ solution. After extraction with $\mathrm{CH}_{2} \mathrm{Cl}_{2}$, the organic phase was dried over anhydrous $\mathrm{Na}_{2} \mathrm{SO}_{4}$ and the solvent was removed under vacuum. The residue was purified by flash chromatography (heptane/EtOAc, 10:1) affording $1.237 \mathrm{~g}$ (100\%) of compound 19 as a colorless oil.

$[\alpha]^{23}$-39.3 (c 1.10, $\mathrm{CHCl}_{3}$ ); Rf 0.34 (heptane/EtOAc, 5:1); IR (film) 2940, 2863, 1611, 1586, $1513,1462,1246,1097,1039 \mathrm{~cm}^{-1} ;{ }^{1} \mathrm{H}$ NMR $\left(500 \mathrm{MHz}, \mathrm{CDCl}_{3}\right) \delta_{\mathrm{H}} 0.80(\mathrm{t}, 3 \mathrm{H}, J=7.4 \mathrm{~Hz})$, $1.05(\mathrm{~m}, 22 \mathrm{H}), 1.19(\mathrm{~d}, 3 \mathrm{H}, J=6.0 \mathrm{~Hz}), 1.44$ (ddd, $1 \mathrm{H}, J=3.3,8.4,13.8 \mathrm{~Hz}), 1.61(\mathrm{~m}, 1 \mathrm{H})$, $1.72(\mathrm{~s}, 3 \mathrm{H}), 2.26(\mathrm{ddd}, 1 \mathrm{H}, J=4.0,9.6,13.8 \mathrm{~Hz}), 2.45(\mathrm{~m}, 1 \mathrm{H}), 3.56(\mathrm{dd}, 1 \mathrm{H}, J=6.6,9.2$ $\mathrm{Hz}), 3.62(\mathrm{dd}, 1 \mathrm{H}, J=5.5,9.4 \mathrm{~Hz}), 3.76(\mathrm{~m}, 1 \mathrm{H}), 3.78(\mathrm{~s}, 3 \mathrm{H}), 3.79(\mathrm{~s}, 3 \mathrm{H}), 4.17(\mathrm{~d}, 1 \mathrm{H}, J=$ $11.7 \mathrm{~Hz}), 4.32$ (dd, 1H, $J=3.3,9.7 \mathrm{~Hz}), 4.42$ (d, 2H, $J=11.5 \mathrm{~Hz}), 4.47$ (d, 1H, $J=11.2 \mathrm{~Hz})$, 
$5.22(\mathrm{~d}, 1 \mathrm{H}, J=10.2 \mathrm{~Hz}), 6.84(\mathrm{~m}, 5 \mathrm{H}), 7.23(\mathrm{~m}, 3 \mathrm{H}) ;{ }^{13} \mathrm{C} \mathrm{NMR}\left(75 \mathrm{MHz}, \mathrm{CDCl}_{3}\right) \delta_{\mathrm{C}} 11.9$, $12.0,12.1,17.9,18.0,18.2,19.6,24.9,41.0,41.9,55.3,55.4,67.1,69.9,70.0,72.5,74.3$, $111.4,113.1,113.9,120.0,121.0,129.3,129.4,131.3,131.9,135.8,140.7,158.9,159.6$; MS (TOF) $\mathrm{m} / \mathrm{z} 621.3\left([\mathrm{M}+\mathrm{Na}]^{+}\right) ; \mathrm{HRMS}(\mathrm{TOF}) \mathrm{m} / \mathrm{z}$ calcd for $\mathrm{C}_{36} \mathrm{H}_{58} \mathrm{O}_{5} \mathrm{NaSi}[\mathrm{M}+\mathrm{Na}]^{+}$ 621.3951, found 621.3963; Anal. calcd for $\mathrm{C}_{36} \mathrm{H}_{58} \mathrm{O}_{5} \mathrm{Si}$ : C 72.19; $\mathrm{H} 9.76$, found $\mathrm{C} 72.17 ; \mathrm{H}$ 9.54 .

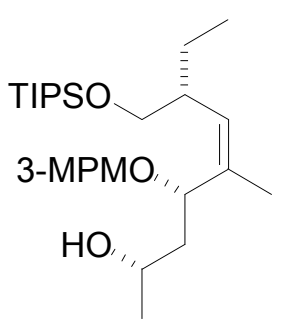

4-(3-Methoxy-benzyloxy)-5-methyl-7-triisopropylsilanyloxymethyl-non-5-en-2-ol (20):

To a solution of compound $19(2.415 \mathrm{~g}, 2.01 \mathrm{mmol})$ in a $\mathrm{CH}_{2} \mathrm{Cl}_{2} / \mathrm{H}_{2} \mathrm{O} 20: 1$ mixture $(25 \mathrm{~mL})$ was added DDQ (456 mg, $2.10 \mathrm{mmol}$ ) at $0{ }^{\circ} \mathrm{C}$. After $60 \mathrm{~min}$ of vigorous stirring, the reaction was poured into $150 \mathrm{~mL}$ of a saturated aqueous $\mathrm{NaHCO}_{3}$ solution. After extraction with $\mathrm{CH}_{2} \mathrm{Cl}_{2}$, the organic phase was dried over anhydrous $\mathrm{Na}_{2} \mathrm{SO}_{4}$, filtered and the solvent was removed under vacuum. The residue was purified by flash chromatography (heptane/EtOAc, 5:1) affording alcohol $\mathbf{2 0}$ along with 4-methoxybenzaldehyde that was totally removed by using a BÜCHI glass oven $\left(120^{\circ} \mathrm{C}, 0.05 \mathrm{mmHg}, 1 \mathrm{~h}\right)$ to give pure alcohol $20(857 \mathrm{mg}, 89 \%)$ as a colorless oil.

$[\alpha]^{23}{ }_{\mathrm{D}}-53.6$ (c 1.20, $\mathrm{CHCl}_{3}$ ); Rf 0.17 (heptane/EtOAc, 5:1); IR (film) 3451, 2941, 2863, 1602 , 1587, 1490, 1461, 1377, $1265 \mathrm{~cm}^{-1} ;{ }^{1} \mathrm{H}$ NMR $\left(500 \mathrm{MHz}, \mathrm{CDCl}_{3}\right) \delta_{\mathrm{H}} 0.81(\mathrm{t}, 3 \mathrm{H}, J=7.5 \mathrm{~Hz})$, $1.05(\mathrm{~m}, 22 \mathrm{H}), 1.16(\mathrm{~d}, 3 \mathrm{H}, J=6.0 \mathrm{~Hz}), 1.46(\mathrm{~d}, 1 \mathrm{H}, J=14.6 \mathrm{~Hz}), 1.62(\mathrm{~m}, 1 \mathrm{H}), 1.73(\mathrm{~s}, 3 \mathrm{H})$, $1.94(\mathrm{dt}, 1 \mathrm{H}, J=10.2,14.6 \mathrm{~Hz}), 2.45(\mathrm{~m}, 1 \mathrm{H}), 3.59$ (ddd, $2 \mathrm{H}, J=5.9,9.3,13.9 \mathrm{~Hz}), 3.69$ (brs, $1 \mathrm{H}), 3.80(\mathrm{~s}, 3 \mathrm{H}), 4.03(\mathrm{~m}, 1 \mathrm{H}), 4.24(\mathrm{~d}, 1 \mathrm{H}, J=11.5 \mathrm{~Hz}), 4.46(\mathrm{~d}, 1 \mathrm{H}, J=11.5 \mathrm{~Hz}), 4.53(\mathrm{~d}$, $1 \mathrm{H}, J=10.3 \mathrm{~Hz}), 5.23(\mathrm{~d}, 1 \mathrm{H}, J=10.3 \mathrm{~Hz}), 6.80-6.89(\mathrm{~m}, 3 \mathrm{H}), 7.23(\mathrm{t}, 1 \mathrm{H}, J=7.7 \mathrm{~Hz}) ;{ }^{13} \mathrm{C}$ $\operatorname{NMR}\left(75 \mathrm{MHz}, \mathrm{CDCl}_{3}\right) \delta_{\mathrm{C}} 12.0,12.1,18.0,18.2,23.7,24.9,42.0,42.5,55.3,67.2,68.2,70.1$, $78.6,113.0,113.5,120.0,129.7,132.3,135.1,139.9,159.9 ; \mathrm{MS}$ (TOF) $\mathrm{m} / \mathrm{z} 501.3$ ([M + $\mathrm{Na}]^{+}$); HRMS (TOF) $\mathrm{m} / \mathrm{z}$ calcd for $\mathrm{C}_{28} \mathrm{H}_{50} \mathrm{O}_{4} \mathrm{NaSi}[\mathrm{M}+\mathrm{Na}]^{+}$501.3376, found 501.3362; Anal. calcd for $\mathrm{C}_{28} \mathrm{H}_{50} \mathrm{O}_{4} \mathrm{Si}$ : C 70.24; $\mathrm{H} 10.53$, found C 70.03; $\mathrm{H} 10.37$.

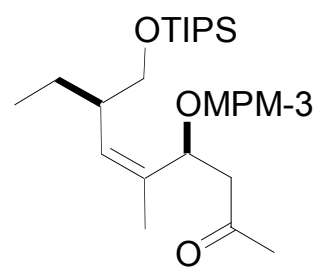

4-(3-Methoxy-benzyloxy)-5,7-dimethyl-8-triisopropylsilanyloxy-oct-5-en-2-one (2):

Alcohol 20 (806 mg, $1.68 \mathrm{mmol})$, activated molecular sieves $4 \AA$ (3.0 g), NMO (296 mg, $2.524 \mathrm{mmol})$ and TPAP (50 mg, $0.059 \mathrm{mmol})$ were stirred in anhydrous $\mathrm{CH}_{2} \mathrm{Cl}_{2}(25 \mathrm{~mL})$ at $\mathrm{rt}$ under argon during $2.5 \mathrm{~h}$. The reaction mixture was filtered and the solvent was removed under vacuum. The residue was purified by flash chromatography (heptane/EtOAc, 10:1) affording $766 \mathrm{mg}(96 \%)$ of ketone $\mathbf{2}$ as a colorless oil.

$[\alpha]^{23}{ }_{\mathrm{D}}-21.3$ (c 1.30, $\mathrm{CHCl}_{3}$ ); Rf 0.25 (heptane/EtOAc, 5:1); IR (film) 2940, 2863, 1716, 1462 , $1264 \mathrm{~cm}^{-1} ;{ }^{1} \mathrm{H}$ NMR $\left(500 \mathrm{MHz}, \mathrm{CDCl}_{3}\right) \delta_{\mathrm{H}} 0.81(\mathrm{t}, 3 \mathrm{H}, J=7.5 \mathrm{~Hz}), 2.12(\mathrm{~s}, 21 \mathrm{H}), 1.10-1.28$ $(\mathrm{m}, 1 \mathrm{H}), 1.62(\mathrm{~m}, 1 \mathrm{H}), 1.73(\mathrm{~s}, 3 \mathrm{H}), 2.17(\mathrm{~s}, 3 \mathrm{H}), 2.32(\mathrm{dd}, 1 \mathrm{H}, J=3.0,15.5 \mathrm{~Hz}), 2.53(\mathrm{~m}$, 
$1 \mathrm{H}), 2.97(\mathrm{dd}, 1 \mathrm{H}, J=9.8,15.5 \mathrm{~Hz}), 3.57(\mathrm{dd}, 1 \mathrm{H}, J=6.2,9.4 \mathrm{~Hz}), 3.61(\mathrm{dd}, 1 \mathrm{H}, J=5.7,9.3$ $\mathrm{Hz}), 3.80(\mathrm{~s}, 3 \mathrm{H}), 4.24(\mathrm{~d}, 1 \mathrm{H}, J=11.5 \mathrm{~Hz}), 4.40(\mathrm{~d}, 1 \mathrm{H}, J=11.5 \mathrm{~Hz}), 4.81(\mathrm{dd}, 1 \mathrm{H}, J=2.9$, $9.8 \mathrm{~Hz}), 5.26(\mathrm{~d}, 1 \mathrm{H}, J=10.3 \mathrm{~Hz}), 6.82(\mathrm{~m}, 3 \mathrm{H}), 7.22(\mathrm{~m}, 1 \mathrm{H}) ;{ }^{13} \mathrm{C} \mathrm{NMR}\left(75 \mathrm{MHz}, \mathrm{CDCl}_{3}\right) \delta_{\mathrm{C}}$ $11.9,12.1,18.0,18.2,24.8,31.2,41.9,48.1,55.3,67.2,70.3,73.7,113.0,113.3,120.0,129.4$, 132.8, 134.5, 140.3, 159.8, 206.8; MS (TOF) m/z $499.3\left([\mathrm{M}+\mathrm{Na}]^{+}\right)$; HRMS (TOF) $\mathrm{m} / \mathrm{z}$ calcd for $\mathrm{C}_{28} \mathrm{H}_{48} \mathrm{O}_{4} \mathrm{NaSi}[\mathrm{M}+\mathrm{Na}]^{+} 499.3220$, found 499.3225 . 

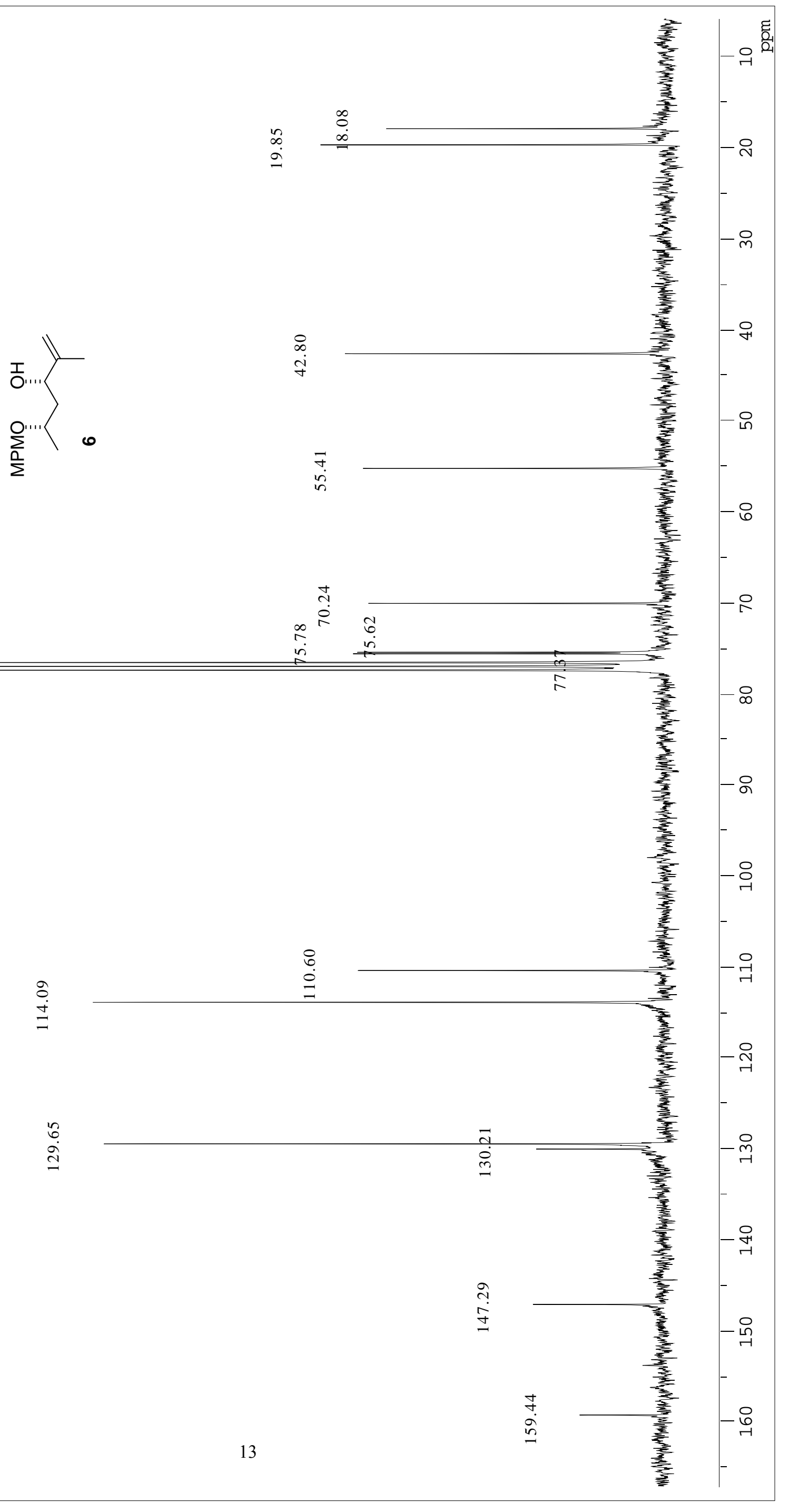
$\stackrel{+}{=}$

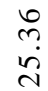

$\stackrel{\infty}{\infty}$

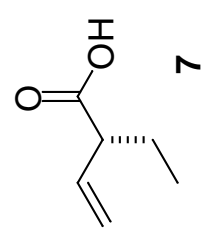

咅咅方

$\stackrel{n}{=}$

- 움

$\underset{v}{\vec{w}}$

당

$\underset{1}{0}$

0
$-\infty$
-1


$-\stackrel{\circ}{\circ}$ 
포
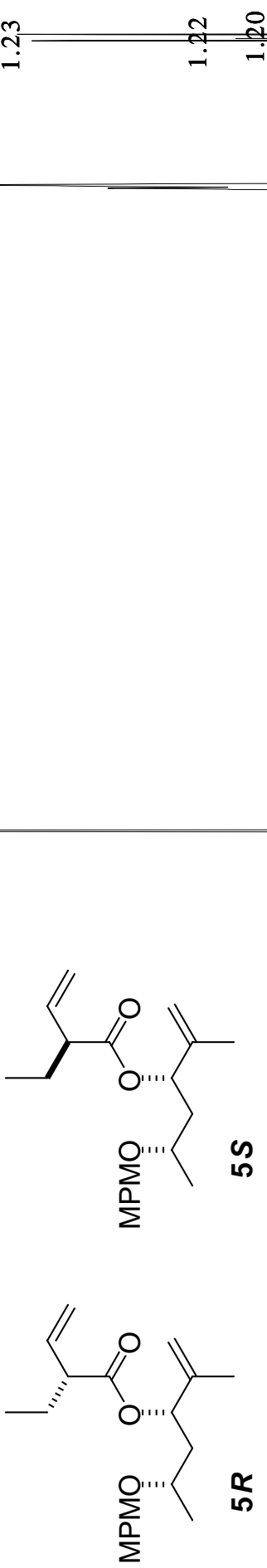

$\frac{\pi}{6}$

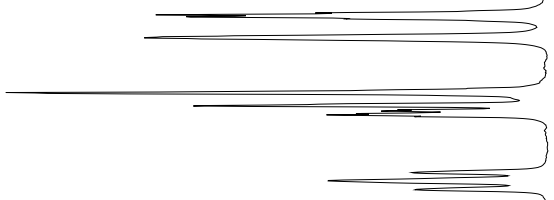

-

-

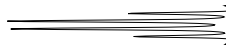

녕요 


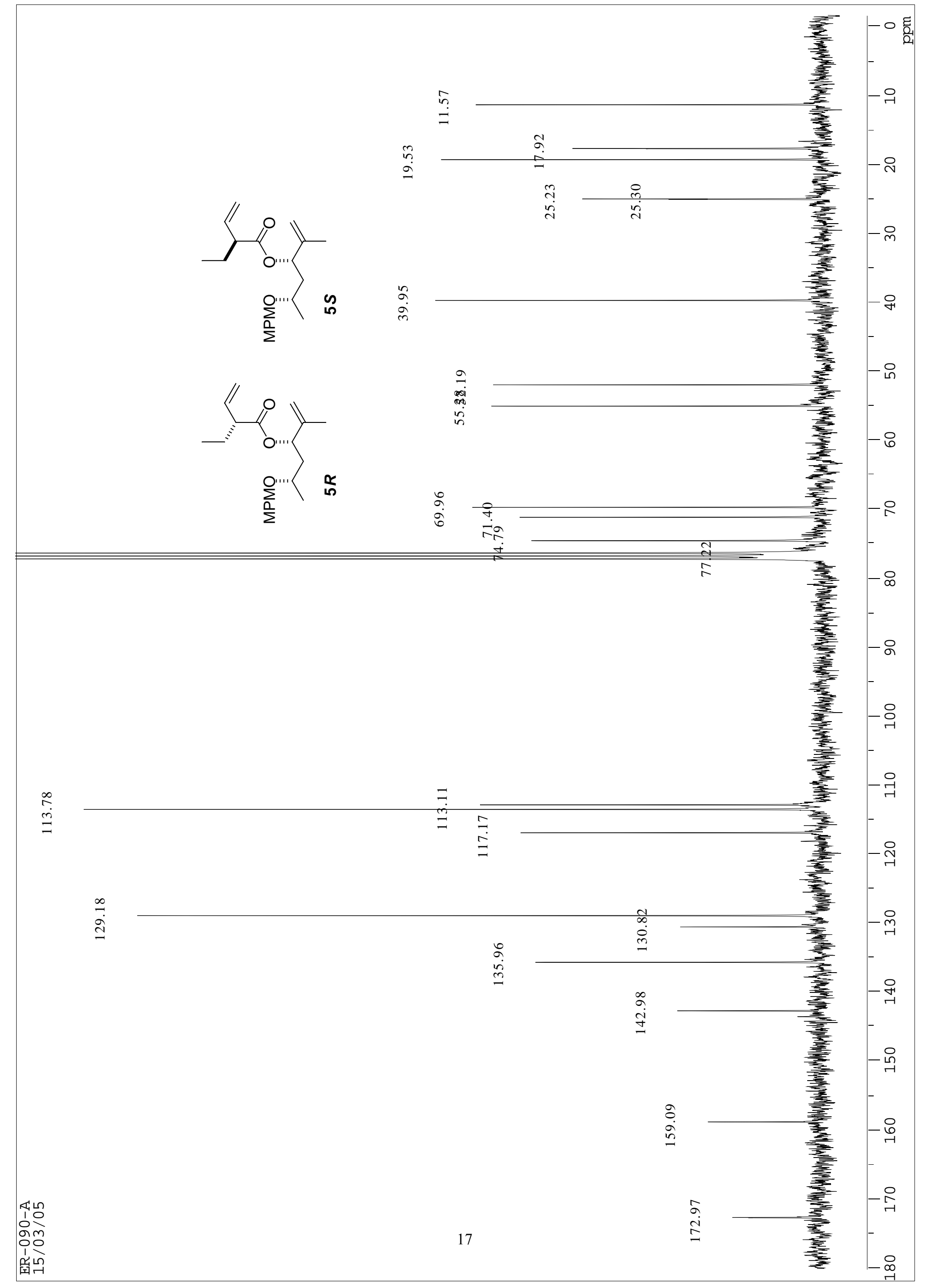




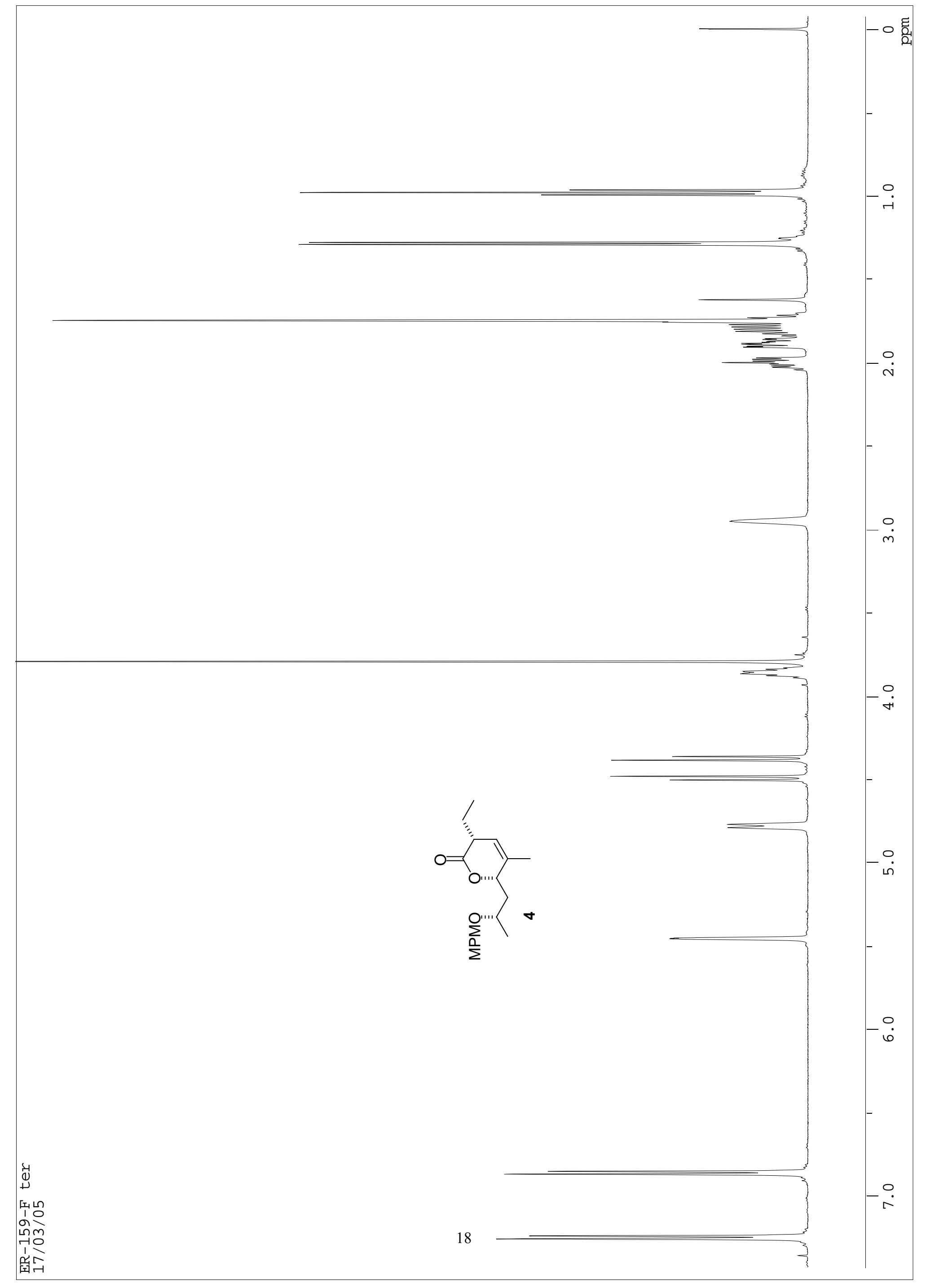




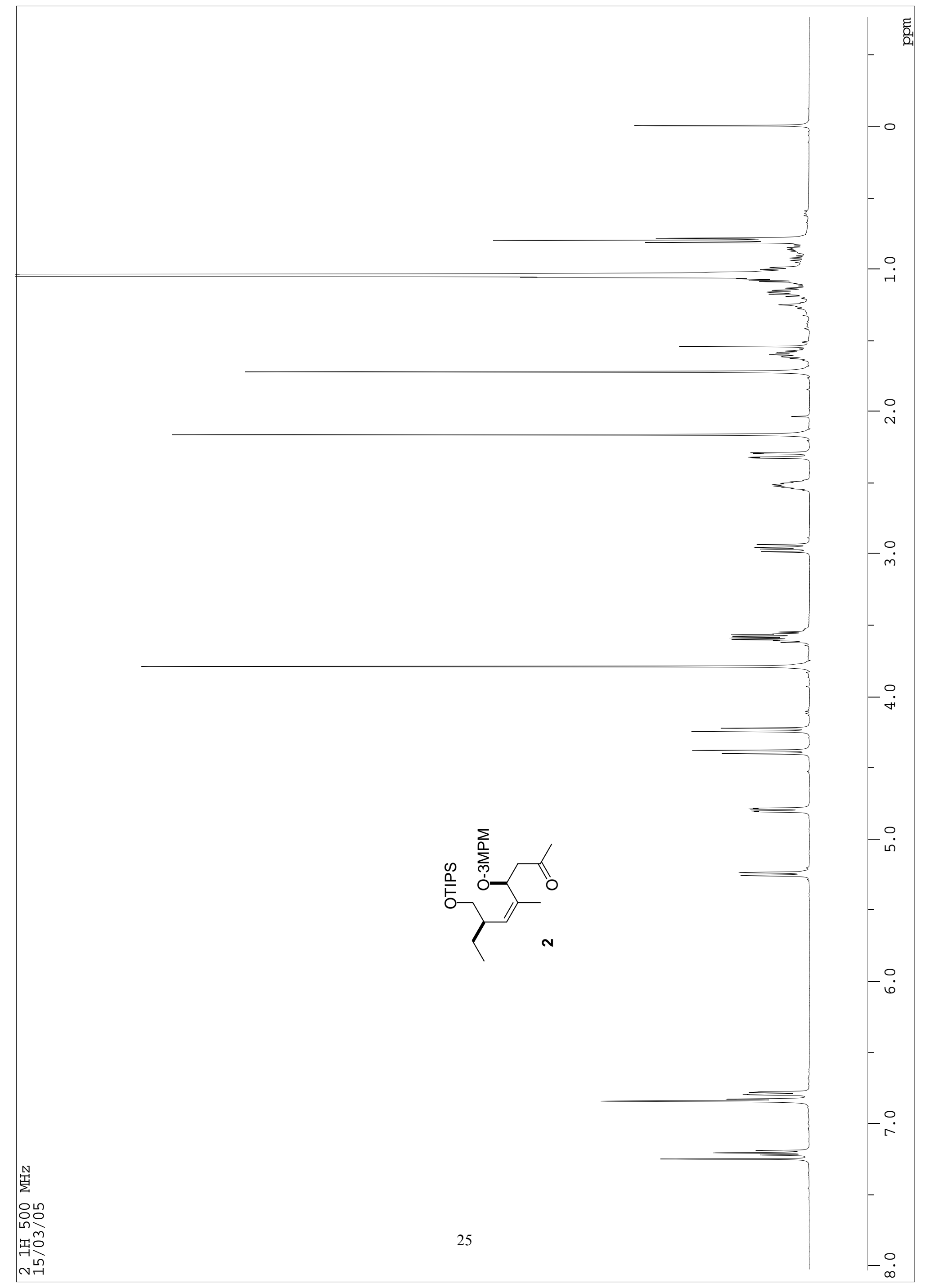


$\stackrel{\infty}{\infty}$

$\stackrel{m}{\stackrel{3}{1}}$

$\sigma$
$\bar{\sigma}$
$\infty$
$\infty$

का

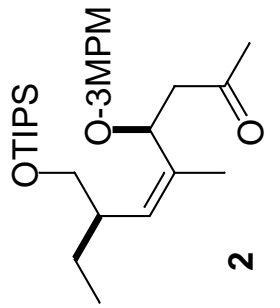

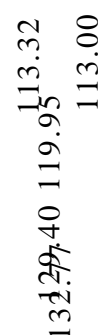

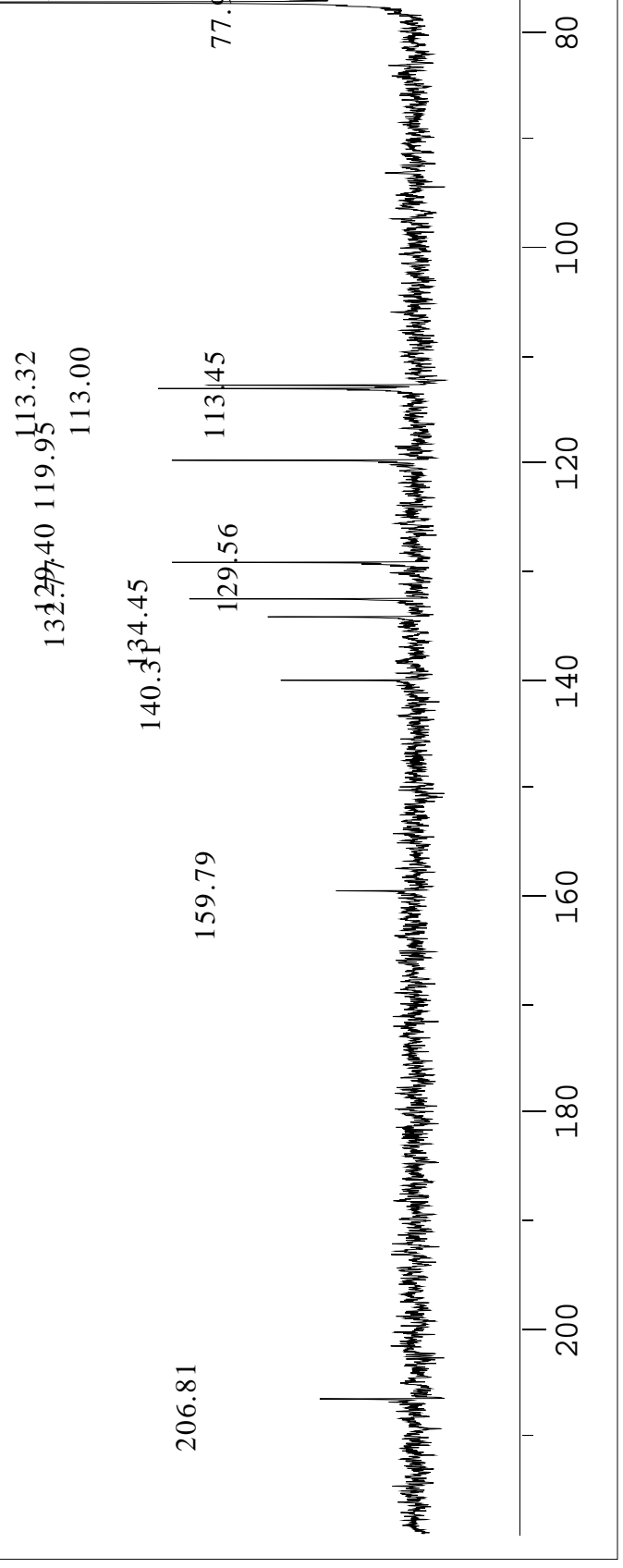

à 